\title{
Ko amar Adonaï : modalités et enjeux du discours prophétique dans le livre de Zacharie (1-8). Analyse littéraire
}

Doctorat de langue et littérature biblique, sous la direction de Hedwige Rouillard-Bonraisin, École pratique des hautes études (EPHE), soutenu le 14 décembre 2011.

\section{Renée Scemama}

\section{(2) OpenEdition}

\section{Journals}

Édition électronique

URL : http://journals.openedition.org/assr/24599

DOI : $10.4000 /$ assr.24599

ISSN : $1777-5825$

Éditeur

Éditions de l'EHESS

\section{Édition imprimée}

Date de publication : 30 décembre 2012

Pagination : 309-358

ISSN : 0335-5985

\section{Référence électronique}

Renée Scemama, « Ko amar Adonaï : modalités et enjeux du discours prophétique dans le livre de Zacharie (1-8). Analyse littéraire », Archives de sciences sociales des religions [En ligne], 160 | octobredécembre 2012, mis en ligne le 14 mars 2013, consulté le 01 mai 2019. URL : http://

journals.openedition.org/assr/24599

Ce document a été généré automatiquement le 1 mai 2019.

(C) Archives de sciences sociales des religions 


\section{Ko amar Adonaï : modalités et enjeux du discours prophétique dans le livre de Zacharie (1-8). Analyse littéraire}

Doctorat de langue et littérature biblique, sous la direction de Hedwige Rouillard-Bonraisin, École pratique des hautes études (EPHE), soutenu le 14 décembre 2011.

\section{Renée Scemama}

1 L'objet de cette étude porte sur la première partie du livre de Zacharie (chap. 1 à 8) comme œuvre «finie », et se propose d'en montrer la cohérence rhétorique, au sens le plus large du terme ${ }^{1}$, en partant du texte massorétique transmis.

2 L'angle d'approche est littéraire et s'appuie sur une étude précise et argumentée du texte hébreu, avec le souci de l'appréhender comme œuvre achevée - fruit de ses effets et de ses procédés - et non comme le produit plus ou moins abouti d'une histoire rédactionnelle longue et compliquée. Le récit des visions et les deux discours qui l'encadrent ne sont pas des œuvres suivies, imputables à un même contexte historique de production ; mais ils forment à eux trois un corpus littéraire relativement indépendant de

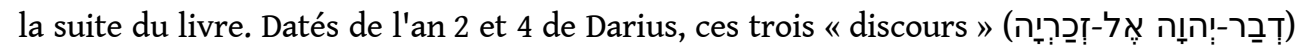
ont été conçus pour être lus dans la continuité les uns des autres et les uns par rapport aux autres, et c'est sur ce principe d'organisation littéraire, qui vaut principe de lecture, que prend appui la présente étude.

3 Adoptant le point de vue du lecteur pour lequel le texte a été organisé comme tel, l'analyse littéraire se propose d'élucider les enjeux de fond qui président à l'organisation interne de chaque discours, et de dégager ceux qui sont susceptibles d'expliquer leur association au sein d'une même unité littéraire. Les trois discours y sont envisagés dans leurs spécificités respectives, et dans le jeu de relation qui les unit - cette seconde question restant tributaire de la première. Du strict point de vue de l'organisation textuelle, ces trois discours forment autant d'unités rhétoriques autonomes et closes sur 
elles-mêmes, c'est-à-dire soumises à une certaine logique interne dont il convient de dégager les principes (hors et indépendamment d'hypothèses relatives à leurs strates rédactionnelles). De fait, ils n'appartiennent pas au même genre littéraire, et les questions que chacun soulève engagent en premier lieu cette spécificité générique, puisque l'un est un « récit », et même un "récit de visions ", tandis que les deux autres sont des "discours prophétiques». Il s'ensuit que les codes qui président à la communication n'y sont pas exactement les mêmes dans les trois ensembles, et que ce trait conditionne en partie la manière dont on peut les appréhender.

En revenant sur ces questions, qui constituent l'apport principal de l'analyse littéraire, nous nous sommes efforcée, à partir des problématiques propres au livre de Zacharie, de rendre compte de l'évolution de la critique biblique contemporaine, tout en montrant la manière dont l'analyse littéraire peut s'articuler aux disciplines historiques.

5 Les trois premiers discours du livre de Zacharie ont fait l'objet d'une attention quelque peu inégale de la part de la critique scientifique contemporaine, et les options qui ont présidé à l'organisation de cette étude témoignent d'une volonté de redonner au premierr et au troisième discours la place qu'ils méritent.

6 Le "récit des visions », qui constitue l'ensemble le plus conséquent de cette partie du livre a suscité de nombreux travaux, et c'est de cette partie du texte qu'il est surtout question lorsqu'on évoque le "proto-Zacharie», c'est-à-dire le corpus littéraire des chapitres 1-8 pour dissiper l'ambiguïté de cette terminologie. Face à ce texte qui continue de fasciner, et dans lequel la critique contemporaine a vu les premières manifestations de la littérature apocalyptique d'époque hellénistique et romaine, les deux discours qui l'encadrent ont parfois fait figure d'œuvres secondaires, sinon mineures. Ils font pourtant partie de cette catégorie de textes que la critique historique envisage désormais comme "l'interprétation, elle-même prophétique, de la tradition prophétique ${ }^{2}-$ un trait qui caractérise déjà le "récit des visions", dont le propos aussi bien que la forme ne s'entendent qu'en référence à d'autres textes. Mais ce qui distingue les premier et troisième discours tient à la nature intensément dialogique du texte et au positionnement tout à fait original de son auteur. Le procédé des « ajouts littéraires » ne consiste pas ici en une intervention isolée ou ponctuelle au sein d'un texte préexistant; il prend la forme de deux « discours prophétiques » de facture extrêmement classique, dont la disposition autour du récit des visions a la propriété particulière d'inverser la problématique initiale, en substituant au thème du retour de Yhwh celui du retour à Yhwh.

7 Ces deux discours fournissent ainsi l'occasion d'observer, au sein d'un texte circonscrit, les stratégies rédactionnelles qui sont à l'œuvre dans la composition des textes et de saisir dans quelle optique travaillent les auteurs bibliques. À terme, ils fournissent aussi de précieux indices sur la manière dont le récit des visions a été lu à date ancienne, puisque leurs auteurs sont pour nous les premiers « lecteurs » qui aient laissé une trace. Du point de vue littéraire, l'ensemble des traits qui les caractérisent participe d'un processus d'écriture dynamique qui prend source dans la lecture des textes et s'explique en partie par elle. À côté des mots "sermon » et "anthologie », qui leur ont parfois été appliqués, c'est celui d'« exégèse » qui nous a semblé leur convenir le mieux.

8 L'analyse de ces deux discours, qui n'en forment en réalité qu'un seul, débouche en effet très rapidement sur des questions liées à la place et à la fonction de cet ensemble textuel, dont la position conclusive, à la fin du corpus prophétique, est à la mesure de ses enjeux. Le premier discours n'est pas une simple introduction au «cycle des visions», et le troisième discours, auquel conduit le raisonnement déployé dans l'exorde, n'a pas pour 
seul objet la question des jeûnes commémoratifs qui lui sert d'amorce. Ce sont l'un et l'autre des œuvres exégétiques de très vaste envergure qui ont été conçues dans une double perspective d'actualisation et de transmission des textes de la tradition scripturaire. Zacharie s'y présente comme celui chez qui s'entend et en qui se résume la parole de tous les "prophètes », et cette posture lui permet, dans le cadre de spéculations liées à la "nouvelle alliance », de revenir sur le délicat problème de la justice divine, dans la continuité de questions évoquées chez Jérémie et Ezéchiel et en réponse au livre des Lamentations.

9 Les problématiques qui sont à l'origine de ces deux discours excèdent donc très largement celles $\mathrm{du}$ " récit des visions » qu'ils encadrent (et dans une certaine mesure décentrent), et leur particularité vient de ce qu'ils réfléchissent, à petite échelle, un pan important d'un corpus scripturaire déjà perçu comme formant un tout. À bien des égards, ces deux discours font donc figure d'œuvre de clôture, et ils témoignent de pratiques exégétiques déjà bien installées qui sont celles dans lesquelles s'engagera toute la littérature ultérieure de commentaires. L'auteur du propos est déjà lui-même dans cette démarche, et les principes qui régissent la communication relèvent eux-mêmes d'une relation de lecteur à lecteur et de textes à textes.

De telles procédures orientent naturellement la réflexion du côté du contexte historique et idéologique dans lequel ils ont été produits. Pour des raisons qui tiennent autant à la forme qu'au contenu, l'hypothèse à laquelle nous sommes parvenue tend à les inscrire dans le contexte qui a vu la clôture du corpus prophétique (Neviim), avec l'idée qu'ils participent étroitement du projet. Mais sur cette question comme sur d'autres, seule la complémentarité des approches est susceptible d'apporter des réponses un peu précises.

11 Du point de vue méthodologique, l'analyse de ces deux discours passe par une claire perception de leur organisation rhétorique, et notamment par une appréciation raisonnée des phénomènes énonciatifs qui structurent le discours (qui dit quoi ? à qui ? dans quel cadre ? et à quelles fins ?). C'est dans cette perspective qu'une importance toute particulière a été accordée aux phénomènes de composition, dans la mesure où la signification d'un texte dépend fondamentalement de l'organisation qu'on lui reconnaît. Il s'est aussi agi d'inverser la démarche qui consiste à sortir immédiatement du texte pour traquer ses références « intertextuelles ", parfois au détriment du propos qu'il développe lui-même. Nous lui avons privilégié celle qui consiste à lire ces discours comme des œuvres singulières, dont les choix d'écriture et de composition participent étroitement de la signification d'ensemble. Elle permet d'approcher au plus près ce qui fonde la spécificité littéraire d'un texte et l'originalité de son écriture. Concernant cet auteur, elles tiennent tout entières dans sa capacité à réécrire sélectivement les autres pour leur faire dire autre chose que ce qu'ils disaient.

12 La partie consacrée au récit des visions ne constitue pas une analyse linéaire et suivie de cette partie du livre. Compte tenu de l'abondance des commentaires dont il a fait l'objet dans la critique contemporaine aussi bien que dans l'exégèse ancienne, il a semblé préférable de traiter sélectivement quelques points particuliers, en prenant appui sur les acquis de la recherche et en gardant pour fil directeur des problématiques littéraires.

13 Parmi celles-ci, la question du contexte nocturne du récit visionnaire - un trait parfaitement inédit dans un "récit de visions » - a permis de revenir sur des questions en rapport avec la place et la fonction qu'occupe le texte. Contrairement à une affirmation souvent reprise, le contexte nocturne du récit de Zacharie ne fait absolument pas partie des «lois du genre», et il n'a pas davantage de rapport avec des realia. Il s'agit d'un 
élément narratif d'ordre symbolique en relation avec les thèmes traités dans cette partie du livre: la reconstruction du temple envisagée comme le "retour de Yhwh" à Jérusalem, au terme des « soixante-dix ans » qu'a duré le châtiment. Dans la continuité de l'histoire narrée dans les livres prophétiques, le récit visionnaire de Zacharie vient faire contrepoint au motif du "prophète privé de visions», qui a fini par désigner métonymiquement le silence de Yhwh. Il marque symboliquement la fin de la « colère » et la reprise du dialogue entre Yhwh et ses "prophètes ». En d'autres termes, ce motif de la nuit ne s'entend qu'en référence aux thèmes traités dans le récit, lesquels s'articulent à des données narratives extérieures au livre lui-même. Le récit des visions n'est pas une œuvre isolée : c'est une suite. Et la manière dont il rend compte de «l'Histoire » ne prend sens que dans le cadre de la narration de l'histoire.

Un autre aspect, en lui-même peu étudié, porte sur l'emploi et le fonctionnement du dialogue, dont les propriétés structurantes contribuent à renouveler les codes de présentation, tout en créant des blancs entre lesquels s'est glissée l'exégèse. Presque exclusivement constitué de paroles rapportées, le récit des visions tend vers une forme d'écriture dramatique qui n'a guère d'équivalent dans la littérature biblique intérieure ou extérieure au corpus. Cette spécificité, qui tient moins à l'introduction du personnage de l'ange qu'au rôle du « naif » que le prophète endosse, en se projetant comme personnage de son propre récit, explique en partie la fortune de sa réception ultérieure. En dépit ou à cause d'une structure très travaillée, le récit des visions est un texte infiniment ouvert qui appelle l'interprétation et que l'interprétation de toute évidence n'épuise pas.

Enfin, les sept études consacrées à l'imagerie des visions ont été conçues comme autant d'articles indépendants organisés autour d'un point précis ("chars et chevaux»; «les myrtes et le gouffre »; « les montagnes d'airain », etc.). Sans prétendre à l'exhaustivité, ils s'efforcent de couvrir, par éclairages successifs, les principaux éléments du récit des visions.

\section{NOTES}

1. M.P. Schmitt et A. Viala, «La Poétique et la Rhétorique sont en relation dialectique : tout texte appartient à un "genre", et relève donc de la Poétique, mais il vise aussi à exercer une action sur son lecteur, et relève de ce fait de la Rhétorique ", Savoir lire, Paris, Didier, 1982, p. 41 et suiv.

2. K. Schmid, «La formation des prophètes postérieurs ", in Th. Römer, Introduction à l'Ancien Testament, Genève, Labor et Fides, 2004, p. 325. 